TI 2012-001/1

Tinbergen Institute Discussion Paper

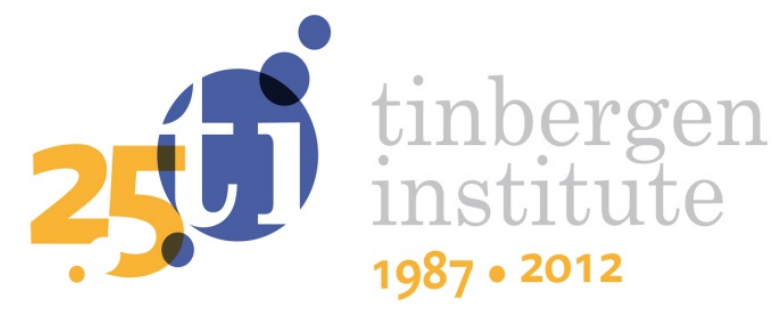

\title{
Multi-Player Agents in Cooperative TU-Games
}

René van den Brink

Chris Dietz

Faculty of Economics and Business Administration, VU University Amsterdam, and Tinbergen Institute. 
Tinbergen Institute is the graduate school and research institute in economics of Erasmus University Rotterdam, the University of Amsterdam and VU University Amsterdam.

More TI discussion papers can be downloaded at http://www.tinbergen.nl

Tinbergen Institute has two locations:

Tinbergen Institute Amsterdam

Gustav Mahlerplein 117

1082 MS Amsterdam

The Netherlands

Tel.: +31(0)205251600

Tinbergen Institute Rotterdam

Burg. Oudlaan 50

3062 PA Rotterdam

The Netherlands

Tel.: +31(0)10 4088900

Fax: $+31(0) 104089031$

Duisenberg school of finance is a collaboration of the Dutch financial sector and universities, with the ambition to support innovative research and offer top quality academic education in core areas of finance.

DSF research papers can be downloaded at: http://www.dsf.nl/

Duisenberg school of finance

Gustav Mahlerplein 117

1082 MS Amsterdam

The Netherlands

Tel.: +31(0)20 5258579 


\title{
Multi-Player Agents in Cooperative TU-games*
}

\author{
René van den Brink ${ }^{\dagger}$ Chris Dietz ${ }^{\ddagger}$
}

December 22, 2011

*This research is financially supported by Netherlands Organization for Scientific Research, NWO grant 400-08-026.

${ }^{\dagger}$ Department of Econometrics and Tinbergen Institute, VU University, De Boelelaan 1105, 1081 HV Amsterdam, The Netherlands. E-mail: jrbrink@feweb.vu.nl.

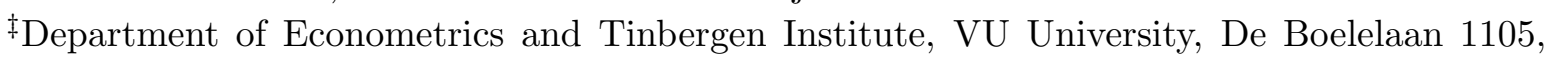
$1081 \mathrm{HV}$ Amsterdam, The Netherlands. E-mail: cdietz@feweb.vu.nl. This author is financially supprted by NWO grant 


\begin{abstract}
A situation in which a finite set of agents can generate certain payoffs by cooperation can be described by a cooperative game with transferable utility (or simply a TU-game) where each agent is represented by one player in the game. In this paper, we assume that one agent can be represented by more than one player. We introduce two solutions for this multi-player agent game model, both being generalizations of the Shapley value for TUgames. The first is the agent-Shapley value and considers the agents in the most unified way in the sense that when an agent enters a coalition then it enters with all its players. The second is the player-Shapley value which takes all players as units, and the payoff of an agent is the sum of the payoffs over all its players.

We provide axiomatic characterizations of these two solutions that differ only in a collusion neutrality axiom. The agent-Shapley value satisfies player collusion neutrality stating that collusion of two players belonging to the same agent does not change the payoff of this agent. On the other hand, the player-Shapley value satisfies agent collusion neutrality stating that after a collusion of two agents, the sum of their payoffs does not change.

After axiomatizing the player- and agent-Shapley values we apply them to airport games. In particular, we distinguish between the costs that depend on the type of airplane that uses a landing strip, and costs that do not depend on this. We argue that for one type of costs the agent-Shapley value is a suitable solution, while for the other type of cost the player-Shapley value is more suitable. Finally, we apply both solutions to voting games.
\end{abstract}

Keywords: Cooperative TU-game, Shapley value, multi-player agent, collusion neutrality, airport games.

JEL code: C71 


\section{Introduction}

A cooperative game with transferable utility, or simply a TU-game, consists of a finite set of players and for every subset (coalition) of players a worth representing the total payoff that the coalition can obtain by cooperating. A (single-valued) solution is a function that assigns to every game a payoff vector which components are the individual payoffs of the players. One of the most applied solutions for cooperative TU-games is the Shapley value (Shapley (1953)).

When modelling an allocation situation as a cooperative TU-game, every decision maker or agent is represented by exactly one element of the player set $N$. However, in many real life situations one agent is represented by various players. For example, one person can be an employee in several firms, thus being a player in each firm. Similar, one person can invest in several firms or belong to the Board of Directors of several firms. One of the main applications of cooperative games in cost allocation is the airport game of Littlechild and Owen (1973), where different airplanes that want to use the same landing strip must pay landing fees that cover the cost of building and maintaining the landing strip, see also Littlechild and Thompson (1977). Although in these airport games the players usually are the airplane movements, i.e. every player represents the landing of one airplane, the real decision making units are the airline companies. So, the airline companies might be considered to be the 'real' decision makers or agents whose players are the landings of any of their airplanes.

In this paper, we generalize the model of a TU-game to deal with situations where each agent can have more than one player. The characteristic function is defined on the set of players, i.e. it are the players who can cooperate and generate worth. Then we introduce two solutions for this model, both being generalizations of the Shapley value for TU-games. The first is the agent-Shapley value and considers the agents in the most unified way. It simply takes an agent with all its players as one unit, and when an agent enters a coalition then it enters with all its players. The other solution is called the player-Shapley value and takes the players as units in the cooperative situation. Here, the payoff of an agent is the sum of the payoffs over all its players.

Interestingly, both these solutions are efficient and satisfy (different) collusion neutrality ${ }^{1}$ properties. This is surprising since van den Brink (2009) showed that requiring efficiency and collusion neutrality at the same time is very restrictive for TU-games. For example, there is no solution for TU-games that satisfies efficiency, collusion neutrality and the null player property. ${ }^{2}$ It turns out that extending the TU-game model to allow agents to be

\footnotetext{
${ }^{1}$ We refer to the collusion neutrality type of axioms that are used by Haller (1994) and Malawski (2002) to characterize the (non-efficient) Banzhaf value for TU-games.

${ }^{2}$ It is also shown that the equal division solution, that distributes $v(N)$ equally over all players, is the
} 
represented by more than one player does allow for collusion neutrality properties that are compatible with efficiency and the null player property. In particular, we provide axiomatizations of the two Shapley type solutions mentioned above that differ only in the collusion neutrality axiom that is used. First, the agent-Shapley value satisfies player collusion neutrality stating that collusion of two players belonging to the same agent does not change the payoff of this agent. On the other hand, the player-Shapley value satisfies agent collusion neutrality stating that after a collusion of two agents, the sum of their payoffs does not change.

After axiomatizing the agent- and player-Shapley values we apply them to airport games and voting games. In particular, for airport games we distinguish between the costs that depend on the size of the airplanes that are using the landing strip, and costs that do not depend on this. We argue that for one type of costs the agent-Shapley value is a suitable solution, while for the other type of cost the player-Shapley value is more suitable. For voting games, the agent Shapley value yields the 'traditional' Shapley value (or Shapley-Shubik index (1954)) for the weighted voting games, often used as a measure assigning voting power to the different parties in parliament. The player-Shapley value simply assigns to every party the number of members in parliament, and is often used to distribute the ministries among the parties that form the government.

The paper is organized as follows. Section 2 contains preliminaries. After presenting the model and the two solutions in Section 3, we provide axiomatic characterizations of these solutions in Section 4. In Section 5 we apply these solutions to sharing costs in airport games and voting games. Section 6 contains concluding remarks. Finally, there is an appendix showing logical independence of the axioms used to characterize the two solutions.

\section{Preliminaries}

A situation in which a finite set of players $N \subset \mathbb{N}$ can generate certain payoffs by cooperation can be described by a cooperative game with transferable utility (or simply a TU-game), being a pair $(N, v)$ where $v: 2^{N} \rightarrow \mathbb{R}$ is a characteristic function on $N$ satisfying $v(\emptyset)=0$. For any coalition $S \subseteq N, v(S) \in \mathbb{R}$ is the worth of coalition $S$, i.e. the members of coalition $S$ can obtain a total payoff of $v(S)$ by agreeing to cooperate. We denote the collection of all characteristic functions on player set $N$ by $\mathcal{G}^{N}$.

A payoff vector for game $(N, v)$ is an $|N|$-dimensional vector $x \in \mathbb{R}^{N}$ assigning a payoff $x_{i} \in \mathbb{R}$ to any player $i \in N$. A (single-valued) solution for TU-games is a function

only solution satisfying efficiency, collusion neutrality and symmetry. 
that assigns a payoff vector to every TU-game. One of the most famous solutions for TU-games is the Shapley value (Shapley (1953)) given by

$$
S h_{i}(N, v)=\sum_{\substack{S \subseteq N \\ i \in S}} \frac{(|N|-|S|) !(|S|-1) !}{|N| !}(v(S)-v(S \backslash\{i\})) \text { for all } i \in N \text {. }
$$

For each nonempty $T \subseteq N, T \neq \emptyset$, the unanimity game $u_{T}$ is given by $u_{T}(S)=1$ if $T \subseteq S$, and $u_{T}(S)=0$ otherwise. It is well-known that the unanimity games form a basis for $\mathcal{G}^{N}$. For every $v \in \mathcal{G}^{N}$ it holds that $v=\sum_{\substack{T \subseteq N \\ T \neq \emptyset}} \Delta_{v}(T) u_{T}$, where $\Delta_{v}(T)=$ $\sum_{S \subseteq T}(-1)^{|T|-|S|} v(S)$ are the Harsanyi dividends, see Harsanyi (1959).

\section{The model and solutions}

In this paper we assume that agents in a cooperative game situation can be represented by more than one player. Therefore, we represent such a multi-player agent game, or shortly multi-player game, by a triple $(N, v, P)$ where $N \subset \mathbb{N}$ is the set of players, $v$ is a characteristic function on the set of players $N$, and $P=\left(P_{1}, \ldots, P_{m}\right)$ is a partition of $N$. Given $P=\left(P_{1}, \ldots, P_{m}\right)$ we denote $M=\{1, \ldots, m\}$. The idea behind the partition $P$ is that every $k \in\{1, \ldots, m\}$ is an agent and $P_{k}$ is its set of players. We denote by $\mathcal{P}^{N}$ the collection of all partitions of $N$, and by $\mathcal{G A}$ the collection of all multi-player cooperative games.

A solution for multi-player games is a function $f: \mathcal{G A} \rightarrow \bigcup_{M \subset \mathrm{N}} \mathbb{R}^{M}$ such that $f(N, v, P) \in \mathbb{R}^{|P|}$ for all $(N, v, P) \in \mathcal{G} \mathcal{A} .^{3}$ In this paper we introduce two solutions that can be considered as extreme cases. In the first solution the agents are considered in the most unified way. It simply takes an agent with all its players as one unit, and when an agent enters a coalition then it enters with all its players. In other words, we consider a game defined on the set of agents $M=\{1, \ldots, m\}$, where each time agent $k \in\{1, \ldots, m\}$ enters a coalition, all players in $P_{k}$ enter the coalition. Therefore, the agent-Shapley value is given by

$$
S_{h}^{a}(N, v, P)=S h\left(M, v^{P}\right)
$$

with $v^{P}(S)=v\left(\bigcup_{k \in S} P_{k}\right)$ for all $S \subseteq M{ }^{4}$

\footnotetext{
${ }^{3}$ Note that the model of a multi-player game is the same as that of a game with coalition structure, see e.g. Aumann and Drèze (1974) and Owen (1977). However, its interpretation and the notion of a solution are different. In terms of multi-player games, a solution for games with coalition structure assigns a payoff to every player, i.e. a payoff vector is a vector in $\mathbb{R}^{N}$, whereas here we assign a payoff to every agent, i.e. a payoff vector is a vector in $\mathbb{R}^{M}$.

${ }^{4}$ Note that $v^{P}$ is the quotient game when $(N, v, P)$ represents a game with coalition structure.
} 
The other solution takes the original game $v: 2^{N} \rightarrow \mathbb{R}$ on the set of players, applies the Shapley value to this game and assigns to every agent the sum of the Shapley values over all its players. Therefore, the player-Shapley value is given by

$$
S h_{k}^{p}(N, v, P)=\sum_{i \in P_{k}} S h_{i}(N, v) \text { for all } k \in M .
$$

In the next section we provide axiomatizations of these two solutions.

\section{Axiomatizations}

We provide axiomatizations of the agent- and player-Shapley values that differ only in a collusion neutrality axiom. The other four axioms are standard for TU-games, but here we present them in terms of multi-player TU-games. The first axiom is efficiency and can be taken directly from the TU-game literature, but adding partition $P$.

Efficiency For every $(N, v, P) \in \mathcal{G} \mathcal{A}$, it holds that $\sum_{k \in M} f_{k}(N, v, P)=v(N)$.

In order to state symmetry, we need to identify symmetric agents in a multi-player game. We say that agents $k, l \in\{1, \ldots m\}$ are symmetric in $(N, v, P)$ if $\left|P_{k}\right|=\left|P_{l}\right|$ (i.e. they have the same number of players), and there exist permutations $\pi^{k}=\left(\pi_{1}^{k}, \ldots, \pi_{\left|P_{k}\right|}^{k}\right)$ on $P_{k}$, and $\pi^{l}=\left(\pi_{1}^{l}, \ldots, \pi_{\left|P_{k}\right|}^{l}\right)$ on $P_{l}$ such that $v\left(S \cup\left\{\pi_{i}^{k}\right\}\right)=v\left(S \cup\left\{\pi_{i}^{l}\right\}\right)$ for all $i \in\left\{1, \ldots,\left|P_{k}\right|\right\}$ and $S \subseteq N \backslash\left\{\pi_{i}^{k}, \pi_{i}^{l}\right\}$ (i.e. the players of agents $k$ and $l$ can be ordered such that two corresponding players are 'symmetric' in game $v$ ).

Symmetry For every $(N, v, P) \in \mathcal{G} \mathcal{A}$, it holds that $f_{k}(N, v, P)=f_{l}(N, v, P)$ whenever $k, l \in M$ are symmetric agents in $(N, v, P)$.

The null player out property of Derks and Haller (1999) states that deleting a null player from a TU-game does not change the payoffs of other players. Here we state a similar property for multi-player games by saying that deleting a null player from any agent, does not change the payoffs. Player $i \in N$ is a null player in game $(N, v)$ if $v(S \cup\{i\})=v(S)$ for all $S \subseteq N \backslash\{i\}$. For game $(N, v)$ we denote the set of null players by $N u l l(v)$. Further, for game $(N, v)$ and $T \subset N$, the restricted game $\left(T, v_{T}\right)$ is given by $v_{T}(S)=v(S)$ for all $S \subseteq T$.

Null player out property For every $(N, v, P) \in \mathcal{G A}$, it holds that $f(N, v, P)=f(N \backslash$ $\left.\{i\}, v_{N \backslash\{i\}},\left(P \backslash\left\{P_{k}\right\}\right) \cup\left\{P_{k} \backslash\{i\}\right\}\right)$ whenever $i \in P_{k}$ is a null player in $(N, v)$ and $\left|P_{k}\right| \geq 2$. 
We generalize strong monotonicity of Young (1985) by saying that whenever all marginal contributions of all players of an agent in game $v$ are at least as much as the corresponding marginal contributions in game $w$, then this agent earns in game $v$ at least as much as in game $w$.

Strong monotonicity For every pair of multi-player games $(N, v, P),(N, w, P) \in \mathcal{G A}$ and agent $k \in\{1, \ldots, m\}$ such that $v(S \cup\{i\})-v(S) \geq w(S \cup\{i\})-w(S)$ for all $i \in P_{k}$ and $S \subseteq N \backslash\{i\}$, it holds that $f_{k}(N, v, P) \geq f_{k}(N, w, P)$.

As mentioned above (and proved later), both the agent- and player Shapley value satisfy these five axioms. They differ with respect to a collusion neutrality axiom they satisfy. Haller (1994) introduced several collusion neutrality properties for TU-games. Such axioms state that the sum of payoffs of two players does not change if they 'collude'. He used these properties to axiomatize the (non-efficient) Banzhaf value. Later, Malawski (2002) showed that several other collusion neutrality properties can be used. ${ }^{5}$ In this paper, we consider two types of collusion, one on the level of the players and one on the level of the agents. Considering the first one, we allow two players of the same agent to collude in the sense of Haller (1994)'s proxy agreement. When player $i \in N$ is going to act as a proxy for player $j \in N \backslash\{i\}$ then instead of $v$ we consider the characteristic function $v_{i j} \in \mathcal{G}^{N}$ given by

$$
v_{i j}(S)= \begin{cases}v(S \backslash\{j\}) & \text { if } i \notin S \\ v(S \cup\{j\}) & \text { if } i \in S .\end{cases}
$$

So, when player $i$ acts as a proxy for another player $j$ then player $j$ becomes a null player, and whenever player $i$ enters a coalition also the contribution of $j$ is added. Similar as shown in van den Brink (2009) for collusion under an association agreement (see Haller (1994)), there is no solution for TU-games that satisfies efficiency, (proxy) collusion neutrality and the null player property. However, it turns out that for multi-player games, there does exist a solution that satisfies all properties mentioned above and the property that collusion of two players belonging to the same agent does not change the payoff of this agent.

Player collusion neutrality For every $(N, v, P) \in \mathcal{G} \mathcal{A}$ with $P=\left\{P_{1}, \ldots, P_{m}\right\} \in \mathcal{P}^{N}$, and $i, j \in P_{k}, k \in\{1, \ldots, m\}$, it holds that $f_{k}(N, v, P)=f_{k}\left(N, v_{i j}, P\right)$.

It turns out that this type of collusion neutrality, together with the above five axioms characterizes the agent-Shapley value. First, we state the following lemma. We call two agents $k, l \in M$ co-dependent in multi-player game $(N, v, P)$ if and only if for every $T \subseteq N$

\footnotetext{
${ }^{5} \mathrm{~A}$ characterization of the Banzhaf value with collusion properties in terms of inequalities can be found in Lehrer (1988).
} 
with $\Delta_{v}(T) \neq 0$ it holds that $\left[P_{k} \cap T \neq \emptyset\right]$ if and only if $\left[P_{l} \cap T \neq \emptyset\right]$. So, two agents are co-dependent if every coalition with nonzero dividend either contains players from agent $i$ as well as from agent $j$, or does not contain any player from these two agents. The next lemma states that a solution satisfying symmetry and the null player out property gives two co-dependent agents $i$ and $j$ the same payoff in game $v$ when both $P_{i}$ and $P_{j}$ contain exactly one non-null player.

Lemma 4.1 Let $f$ be a solution satisfying symmetry and the null player out property, and let $i, j \in M$ be two co-dependent agents such that $\left|P_{k} \backslash N u l l(v)\right|=\left|P_{l} \backslash N u l l(v)\right|=1$. Then $f_{k}(N, v, P)=f_{l}(N, v, P)$.

\section{ProOF}

Suppose that solution $f$ satisfies symmetry and the null player out property, and let $k, l \in$ $M$ be two co-dependent agents such that $\left|P_{k} \backslash N u l l(v)\right|=\left|P_{l} \backslash N u l l(v)\right|=1$. The null player out property implies that $f(N, v, P)=f\left(N, v, P^{\prime}\right)$, where $P^{\prime}=\left(P_{1}^{\prime}, \ldots, P_{m}^{\prime}\right)$ with $P_{h}^{\prime}=P_{h} \backslash N u l l(v)$ if $h \in\{k, l\}$, and $P_{h}^{\prime}=P_{h}$ otherwise. Since $i$ and $j$ are also co-dependent in $\left(N, v, P^{\prime}\right)$, by symmetry $f_{k}\left(N, v, P^{\prime}\right)=f_{l}\left(N, v, P^{\prime}\right)$, and thus $f_{k}(N, v, P)=f_{l}(N, v, P)$.

Now we state our axiomatization of the agent-Shapley value.

Theorem 4.2 A solution $f$ for multi-player games is equal to the agent-Shapley value if and only if it satisfies efficiency, strong monotonicity, symmetry, the null player out property and player collusion neutrality.

\section{ProOF}

It is straightforward to verify that the agent-Shapley value satisfies efficiency, strong monotonicity, symmetry and the null player out property. Player collusion neutrality follows since $v^{P}=\left(v_{i j}\right)^{P}$ if $i, j \in P_{k}$ for some $k \in\{1, \ldots, m\}$.

To show uniqueness, suppose that solution $f$ satisfies these five axioms.

Recall that every game $v$ can be written as a (unique) linear combination of unanimity games by $v=\sum_{\substack{T \subseteq N \\ T \neq \emptyset}} \Delta_{v}(T) u_{T}$. Let $D(N, v)=\left\{T \subseteq N \mid \Delta_{v}(T) \neq 0\right\}$, and $d(N, v)=$ $|D(N, v)|$. Similar as in Young (1985), we prove uniqueness by induction on $d(N, v)$.

If $d(N, v)=0$ (i.e. $(N, v)$ is a null game given by $v(S)=0$ for all $S \subseteq N$ ) then by the null player out property we have that $f_{k}(N, v, P)=f_{k}\left(N, v, P^{\prime}\right)$ where $P^{\prime}=\left(P_{1}^{\prime}, \ldots, P_{m}^{\prime}\right)$ with $P_{k}^{\prime} \subseteq P_{k}$ such that $\left|P_{k}^{\prime}\right|=1$ for all $k \in M$. Since all agents are symmetric in $(N, v, P)$, symmetry implies that all $f_{k}\left(N, v, P^{\prime}\right)$ are equal, and thus all $f_{k}(N, v, P)$ are equal. By efficiency, $f_{k}(N, v, P)=0$ for all $k \in M$. 
Proceeding by induction, assume that $f(N, w, P)$ is uniquely determined whenever $d(N, w)<$ $d(N, v)$. Define $R(N, v, P)=\left\{k \in M \mid P_{k} \cap T \neq \emptyset\right.$ for all $\left.T \in D(N, v)\right\}$ as the set of agents that have at least one player in every unanimity coalition with nonzero dividend.

If $k \in M \backslash R(N, v, P)$ then there exists a $T \in D(N, v)$ with $T \cap P_{k}=\emptyset$. Strong monotonicity then implies that $f_{k}(N, v, P)=f_{k}\left(N, v-\Delta_{v}(T) u_{T}, P\right)$, which is uniquely determined by the induction hypothesis.

To determine the payoffs of the agents in $R(N, v, P)$, define $Q(N, v, P)=\{k \in R(N, v, P) \mid$ $\left.\left|P_{k} \backslash N \operatorname{ull}(v)\right|>1\right\}$ as the set of agents in $R(N, v, P)$ that have more than one non-null player, and let $q(N, v, P)=|Q(N, v, P)|$. We determine $f_{k}(N, v, P), k \in R(N, v, P)$, by performing a second induction on $q(N, v, P)$.

If $q(N, v, P)=0$, i.e. all agents have at most one non-null player who is in every $T \in$ $D(N, v)$, then any two players in $R(N, v, P)$ are co-dependent, and thus by Lemma 4.1 there exists a $c^{*} \in \mathbb{R}$ such that $f_{k}(N, v, P)=c^{*}$ for all $k \in R(N, v, P)$. Efficiency then uniquely determines $c^{*}$, and thus $f_{k}(N, v, P)$ for all $k \in R(N, v, P)$.

Proceeding by induction, suppose that $f(N, w, P)$ is uniquely determined whenever $q(N, w, P)<$ $q(N, v, P)$. Take $k \in Q(N, v, P)$ and $i, j \in P_{k} \backslash N u l l(v), i \neq j$. (Note that such $i$ and $j$ exist since $k \in Q(N, v, P)$.) Collusion neutrality implies that $f_{k}(N, v, P)=f_{k}\left(N, v_{i j}, P\right)$. Note that $j$ is a null player in $v_{i j}$. Repeated application of player collusion neutrality in this way, yields that $f_{k}(N, v, P)=f_{k}\left(N, v^{i}, P\right)$ with, for some $i \in P_{k}$, the characteristic function $v^{i}$ given by $v^{i}=v+\sum_{T \cap C_{T} \neq \bar{P}_{k} \neq \emptyset} \Delta_{v}(T)\left(u_{\left(T \backslash P_{k}\right) \cup\{i\}}-u_{T}\right)$. Note that all $j \in P_{k} \backslash\{i\}$ are null players in $v^{i}$. Since, $j \in N \backslash P_{k}$ is a null player in $v^{i}$ if and only if it is a null player in $v$, we have that $q\left(N, v^{i}, P\right)=q(N, v, P)-1$, and the payoff $f_{k}(N, v, P)=f_{k}\left(N, v^{i}, P\right)$ is determined by the induction hypothesis.

Finally, all $i \in R(N, v, P) \backslash Q(N, v, P)$ are symmetric in $(N, v, \bar{P})$ where $\bar{P}=\left\{\bar{P}_{1}, \ldots, \bar{P}_{m}\right\}$ is such that $\bar{P}_{k}=P_{k} \backslash N u l l(v)$ if $k \in R(N, v, P) \backslash Q(N, v, P)$, and $\bar{P}_{k}=P_{k}$ otherwise. Thus, by symmetry there exists a $c^{*} \in \mathbb{R}$ such that $f_{k}(N, v, \bar{P})=c^{*}$ for all $k \in R(N, v, P) \backslash$ $Q(N, v, P)$. Since all the other payoffs are determined, efficiency determines $c^{*}$, and thus $f_{k}(N, v, \bar{P})$ for all $k \in R(N, v, P) \backslash Q(N, v, P)$. But then the null player out property determines $f_{k}(N, v, P), k \in R(N, v, P) \backslash Q(N, v, P)$.

Logical independence of the axioms in Theorem 4.2 is shown in the appendix of the paper.

Besides collusion between players of one agent, one might also consider collusion between agents. In this case we do not change the game, but merge the two agents by replacing them with one agent who 'controls' all players of the two original agents together. So, a collusion between agents $k, l \in M=\{1, \ldots, m\}$ can be described by merging the sets 
of players of these two agents, i.e. by considering the partition

$$
P^{k l}=\left(P \backslash\left\{P_{k}, P_{l}\right\}\right) \cup\left\{P_{k} \cup P_{l}\right\} .
$$

Assuming without loss of generality that $k<l$, we label the elements in $P^{k l}$ such that $P_{k}^{k l}=P_{k} \cup P_{l}$, and $P_{h}^{k l}=P_{h}$ for all $h \in M \backslash\{k, l\}$.

Agent collusion Neutrality For every $(N, v, P) \in \mathcal{G A}$, it holds that $f_{k}(N, v, P)+f_{l}(N, v, P)=$ $f_{k}\left(N, v, P^{k l}\right)$.

Replacing in Theorem 4.2 player collusion neutrality by this agent collusion neutrality characterizes the player-Shapley value. Moreover, in this case we can do without the null player out property.

Theorem 4.3 A solution $f$ is equal to the player-Shapley value if and only if it satisfies efficiency, strong monotonicity, symmetry and agent collusion neutrality.

\section{PROOF}

It is straightforward to verify that the player-Shapley value satisfies efficiency, strong monotonicity and symmetry. Agent collusion neutrality follows since $P_{k} \cap P_{l}=\emptyset$, and thus $\left\{P_{k}, P_{l}\right\}$ is a partition of $P_{k} \cup P_{l}$, so $\sum_{i \in P_{k}} S h_{i}(N, v)+\sum_{i \in P_{l}} S h_{i}(N, v)=\sum_{i \in P_{k} \cup P_{l}} S h_{i}(N, v)$.

Now, suppose that solution $f$ satisfies these four axioms. To prove uniqueness, we perform induction on the number of agents with more than one player. as in the proof of Theorem 4.2 , we define $D(N, v)=\left\{T \subseteq N \mid \Delta_{v}(T) \neq 0\right\}, d(N, v)=|D(N, v)|$, and $R(N, v, P)=$ $\left\{k \in M \mid P_{k} \cap T \neq \emptyset\right.$ for all $\left.\left.T \in D(N, v)\right\}\right)$.

Further, let $H(N, P)=\left\{k \in M|| P_{k} \mid \geq 2\right\}$ be the set of agents having at least two players, and $h(N, P)=|H(N, P)|$.

First, suppose that $h(N, P)=0$, i.e. $\left|P_{k}\right|=1$ for all $k \in M$. Then the proof is similar to that of Young (1985). We perform a second induction on $d(N, v)$. If $d(N, v)=0$ then by symmetry and efficiency $f_{k}(N, v, P)=0$ for all $k \in M$. Proceeding by induction, assume that $f(N, w, P)$ is uniquely determined whenever $d(N, w)<d(N, v)$. If $k \in M \backslash$ $R(N, v, P)$ then there is a $T \subseteq N \backslash P_{k}$ with $\Delta_{v}(T) \neq 0$. But then, $f_{k}(N, v, P)=f_{k}(N, v-$ $\left.\Delta_{v}(T) u_{T}, P\right)$ by strong monotonicity. Thus, $f_{k}(N, v, P)$ is uniquely determined by the induction hypothesis. Since all $k \in R(N, v, P)$ are symmetric, their payoffs are then determined by symmetry and efficiency.

Proceeding by induction, suppose that $f\left(N, v, P^{\prime}\right)$ is determined whenever $h\left(N, P^{\prime}\right)<$ $h(N, P)$. For $k \in H(N, P)$, with $P_{k}=\left\{k_{1}, \ldots, k_{p}\right\}$, consider $\widetilde{P}=\left(\widetilde{P}_{1}, \ldots, \widetilde{P}_{k-1}, \widetilde{P}_{k_{1}}, \ldots, \widetilde{P}_{k_{p}}, \widetilde{P}_{k+1}, \ldots, \widetilde{P}_{m}\right)$ with $\widetilde{P}_{h}=P_{h}$ for all $h \in M \backslash\{k\}$, and $\widetilde{P}_{k_{i}}=\left\{k_{i}\right\}$ for $k_{i} \in P_{k}$. Since $h(N, \widetilde{P})<h(N, P)-1$, the induction hypothesis implies 
that $f(N, v, \widetilde{P})$ is uniquely determined. But then agent collusion neutrality implies that $f_{k}(N, v, P)=\sum_{i=1}^{p} f_{k_{i}}(N, v, \widetilde{P})$ is determined.

For agents $k \in M \backslash H(N, P)$, i.e. the agents with one player, the proof goes again similar as Young (1985)'s or the proof above for the case $h(N, P)=0$. We again perform a second induction on $d(N, v)$. If $d(N, v)=0$ then all agents in $M \backslash H(N, P)$ are symmetric, and since we already determined the payoffs of the agents in $H(N, P)$, efficiency and symmetry determine the payoffs of the players in $M \backslash H(N, P)$. Proceeding by induction, assume that $f_{k}(N, w, P)$ is uniquely determined whenever $d(N, w)<d(N, v)$. If $k \in$ $M \backslash(H(N, P) \cup R(N, v, P))$ then there is a $T \subseteq N \backslash P_{k}$ with $\Delta_{v}(T) \neq 0$. But then, $f_{k}(N, v, P)=f_{k}\left(N, v-\Delta_{v}(T) u_{T}, P\right)$ by strong monotonicity. Thus, $f_{k}(N, v, P)$ is uniquely determined by the induction hypothesis. Since all $k \in(M \backslash H(N, P)) \cap R(N, v, P)$ are symmetric, and all the other payoffs are determined, also their payoffs are determined by symmetry and efficiency.

Logical independence of the axioms in Theorem 4.3 is also shown in the appendix of the paper.

As mentioned before, van den Brink (2009) shows that there is no solution for TUgames that satisfies efficiency, collusion neutrality and the null player out property. Above we saw that for multi-player games, the agent-Shapley value and player-Shapley value both satisfy efficiency and a different collusion neutrality. Moreover, both solutions satisfy the null agent property which generalizes the null player property. In order to state this property, we need to specify what is a null agent in a multi-player game. We say that an agent is a null agent if all its players are null players in $v$, i.e. solution $f$ satisfies the null agent property if for every $(N, v, P) \in \mathcal{G} \mathcal{A}$, it holds that $f_{k}(N, v, P)=0$ whenever $k \in M$ is a null agent in $(N, v, P)$.

We remark that in both Theorem 4.2 as well as in Theorem 4.3, the player set $N$ is fixed. Concerning the agents, in Theorem 4.2 the set of agents is fixed, but Theorem 4.3 is stated for a variable agent set. We saw that, given the other axioms, in that case the null player out property is not needed anymore. Similar, we could state player collusion neutrality for a variable player set by requiring that for every $(N, v, P) \in \mathcal{G A}$ with $P=$ $\left\{P_{1}, \ldots, P_{m}\right\} \in \mathcal{P}^{N}$, and $i, j \in P_{k}, i \neq j, k \in\{1, \ldots, m\}$, it holds that $f_{k}(N, v, P)=$ $f_{k}\left(N \backslash\{j\},\left(v_{i j}\right)_{N \backslash\{j\}}, P^{-j}\right)$, where $P_{k}^{-j}=P_{k} \backslash\{j\}$, and $P_{l}^{-j}=P_{l}$ for all $l \in M \backslash\{k\}$. Similar as the proof of Theorem 4.2, it can be shown that in that case the null player out property is not needed anymore, and the agent-Shapley value is characterized by the remaining four axioms. Thus, the agent-Shapley value and the player-Shapley value are characterized by comparable axioms in the sense that they differ only in the collusion 
neutrality property that is used.

\section{Applications}

\subsection{Airport games}

Airport games are introduced by Littlechild and Owen (1973) to allocate the building and maintenance costs of airport landing strips, see also Littlechild and Thompson (1977). ${ }^{6}$ Suppose there are $n$ airplanes that want to use the same landing strip. ${ }^{7}$ The $n$ airplanes are different and therefore need landing strips of different size. Assume that the airplanes are labeled so that the cost of building or maintaining a landing strip for airplane $i \in\{1, \ldots, n\}$ is given by $c_{i}$ satisfying $0 \leq c_{1} \leq c_{2} \leq \ldots \leq c_{n}$. The corresponding airport game (see Littlechild and Owen (1973)) is a cost game $(N, v)$ with the set of airplanes as the set of players and the characteristic function given by $v(S)=\max _{i \in S} c_{i}$ for all $S \subseteq N$. So, the total cost of the landing strip that can handle all the $n$ airplanes is determined by the most expensive airplane (in terms of landing strip). For simplicity, we assume that the larger the airplane the larger (and more expensive) is the landing strip it needs, and the larger is its contribution to the maintenance costs. Applying the Shapley value to allocate the total cost over the airplanes one arrives at the famous formula

$$
S h_{i}(v)=\sum_{j=1}^{i} \frac{c_{j}-c_{j-1}}{n-j+1} \text { for all } i \in N
$$

where $c_{0}=0$, see Littlechild and Owen (1973).

Instead of allocating costs over the airplanes, using multi-player games we can allocate the costs over the airline companies. Consider the multi-player game $(N, v, P)$ where $N=\{1, \ldots, n\}$ is the set of airplanes, $v: 2^{N} \rightarrow \mathbb{R}$ is the airport (cost) game described above, and $P=\left\{P_{1}, \ldots, P_{m}\right\}$ is the partition of the set of airplanes into the $m$ airlines, such that $P_{k}$ is the set of airplanes owned by airline $k \in\{1, \ldots, m\}$. Applying the agentShapley value, the cost allocation will be as if applying the Shapley value to the associated game

$$
v^{P}(S)=v\left(\bigcup_{k \in S} P_{k}\right)=\max _{i \in \bigcup_{k \in S} P_{k}} c_{i} \text { for all } S \subseteq M .
$$

\footnotetext{
${ }^{6}$ Although we do not discuss them further, we remark that the class of airport games is equivalent to the class of dual auction games (see Graham, Marshall and Richard (1990)) and DR-polluted river games (see Ni and Wang (2007)).

${ }^{7}$ Instead of airplanes, in an airport game every airplane landing is a player but for convenience we simply call these airplanes.
} 
So, the cost of a coalition of airlines is fully determined by the largest airplane among the airlines in the coalition. In particular, $v^{P}(\{k\})=\max _{i \in P_{k}} c_{i}$ is the cost of the largest airplane of airline $k$. Each time an airline enters a coalition, its marginal contribution is fully determined by its largest airplane. Taking the Shapley value of this game, we allocate the costs among the airlines in a similar spirit as the Shapley value for airport games allocates the cost over the airplanes. So, the agent-Shapley value seems reasonable to apply when allocating costs that are fully determined by the largest airplane that uses the landing strip.

However, when we allocate maintenance costs that do not depend on the length of the landing strip needed for the largest airplane, the agent-Shapley value is not suitable. For these costs we can apply the player-Shapley value which first determines fees by the Shapley value for each airplane and after that, lets every airline pay the sum of the fees allocated to all its airplanes.

Example 5.1 Consider a situation with two airlines where airline 1 owns two airplanes, and airline 2 owns three airplanes. Suppose airline 1 owns airplanes 2 and 5, while airline 2 owns airplanes 1,3 and 4 . Consider the $\operatorname{costs} c_{i}=i$ for $i \in N=\{1, \ldots, 5\}$. If these are the building costs that depend only on the size of the largest airplane then the costs can be allocated using the agent-Shapley value. The associated game $v^{P}$ is given by $v^{P}(\{1\})=5, v^{P}(\{2\})=4$ and $v^{P}(\{1,2\})=5$, yielding agent-Shapley value $S h^{a}(N, v, P)=$ $\operatorname{Sh}\left(N, v^{P}\right)=(3,2)$. However, if these are maintenance costs that depend more on the use of the landing strip than on the size of the largest airplane, then using the player-Shapley value we obtain as Shapley values of the airplanes $S h(N, v)=\frac{1}{60}(12,27,47,77,137)$. Adding the costs over each airlines own airplanes yields the player-Shapley value $\operatorname{Sh}^{p}(N, v, P)=$ $\frac{1}{60}(149,151)$. So, according to the player-Shapley value agent 2 pays more than agent 1 , while according to the agent-Shapley value agent 1 pays more than agent 2 .

Considering the interpretation of the two collusion neutrality axioms to airport games, player collusion neutrality implies that when an airline does not use anymore one of its airplanes but its largest airplane, then its share in the costs does not change. This seems reasonable when allocating costs that are fully determined by the costs for the largest airplane. On the other hand, agent collusion neutrality implies that when two airlines merge in such a way that their 'fleet' consists of exactly the two sets of airplanes that were owned by the separate airlines before merging, then the total share in the costs of the new merged airline equals the sum of the shares in the cost of the two previous airlines. This seems reasonable when one has to allocate costs that depend on all landings. 


\subsection{Voting power}

Measuring power in political bodies is often done by applying TU-game solutions to simple games representing the possibilities of political parties to form majority coalitions. A TUgame is called simple if $v(S) \in\{0,1\}$ for every $S \subseteq N$. In such a game a coalition is called winning when its worth equals one, otherwise (i.e. when its worth is zero), it is called losing. Usually the player set in such games is formed by the political parties that belong to the voting body (and not by the individual members of, say, the parliament). A special class of simple games that are often used to represent political bodies are weighted majority voting games. These games describe situations where a coalition of parties is winning if and only if this coalition in total has a number of seats (or votes) that is at least equal to some quota, which is often taken to be higher than half of the number of seats.

Formally, a weighted voting game is given by a tuple $\left(M ; s_{1}, \ldots, s_{n} ; q\right)$, where $s_{i} \in \mathbb{N}$ is the number of votes of player $i, i \in M$, and the quota $q \in \mathbb{N}$ is the number of votes needed to win. The corresponding simple game $(M, v)$ is given by $v(S)=1$ when $\sum_{i \in S} s_{i} \geq q$ ( $S$ is winning) and $v(S)=0$ otherwise. A simple game is proper if $v(S)=1$ implies that $v(M \backslash S)=0$. So, for a weighted majority voting game this means that $q>\frac{1}{2} \sum_{i \in M} s_{i}$.

One can use voting power measures or indices, such as the Banzhaf index (see Banzhaf (1965)) or the Shapley-Shubik index (see Shapley and Shubik (1954)), to measure the voting power of the political parties in a parliament. The Shapley-Shubik index is obtained by applying the Shapley value to the associated simple voting game. Both indices are based on the marginal contributions of players in voting games. Note that the marginal contribution of a player to any coalition in a weighted majority voting game is either zero or one. It equals one if and only if the player turns a losing coalition into a winning one. When the marginal contribution of player $i$ to coalition $S$ equals one, then $S$ is called a swing for player $i$.

Although the power of political parties depends heavily on the swings of that party, usually when a majority coalition forms a government, the parties in that coalition divide the number of ministries among them proportional to the number of seats each party has, and not using the 'real' voting power of the parties. So, it seems that both the Banzhaf and Shapley-Shubik voting power indices as well as the seat distribution among parties plays a role in the formation of a government. The player- and agent-Shapley values can be used to obtain both.

Consider the multi-player game $(N, v, P)$ where the set $N$ is the set of members (seats) of parliament, $v$ is the characteristic function defined on the set of members of parliament, and $P=\left(P_{1}, \ldots, P_{m}\right)$ is the partition of the members $N$ into the different political parties. So, the members in $P_{k}$ are exactly those that belong to party $k \in$ $\{1, \ldots, m\}$. Notice that $v(S)=1$ if and only if $|S| \geq q$, even if $S$ contains members of 
different parties. So, all members (players) are symmetric, and thus applying the Shapley value yields $S h_{i}(N, v)=\frac{1}{n}$ for all $i \in N$. The player-Shapley value of party (agent) $k \in M$ equals the fraction of seats of party $k: S h_{k}^{p}(N, v, P)=\sum_{i \in P_{k}} S h_{i}(N, v)=\frac{s_{k}}{|N|}$ where $s_{k}=\left|P_{k}\right|$, yields the seat distribution.

On the other hand, applying the agent-Shapley value $S_{h}^{a}(N, v, P)=S h\left(P, v^{P}\right)$, we see that $v^{P}$ is similar to a 'standard' voting game on the set of parties, and thus this yields the Shapley-Shubik index of the standard voting game.

Example 5.2 Consider the weighted majority voting game $\left(M ; s_{1}, \ldots, s_{n} ; q\right)$ with $M=$ $\{1,2,3\}, s_{1}=20, s_{2}=s_{3}=40$ and $q=51$. So, there are 100 members of parliament who are divided among three parties, and decisions are made by majority voting. Assuming the seats to be labeled such that each party has a consecutive set of seats, this can be modelled by multi-player game $(N, v, P)$ with $N=\{1, \ldots, 100\}, P=\left(P_{1}, P_{2}, P_{3}\right)$ with $P_{1}=\{1, \ldots, 20\}, P_{2}=\{21, \ldots, 60\}$ and $P_{3}=\{61, \ldots, 100\}$. Considering voting power, the game $v^{P}$ is given by $v^{P}(S)=1$ if $S \subseteq M$ with $|S| \geq 2$, and $v^{P}(S)=0$ otherwise. Then all agents are symmetric in $v^{P}$, and the agent-Shapley value $\operatorname{Sh}^{a}(N, v, P)=\operatorname{Sh}\left(M, v^{P}\right)=$ $\left(\frac{1}{3}, \frac{1}{3}, \frac{1}{3}\right)$ which equals the Shapley-Shubik index and expresses the fact that each party is equally powerfull in turning losing coalitions into winning ones. However, when parties form a majority coalition then the ministries are usually distributed proportional to the seat distribution which is obtained as the player-Shapley value $\operatorname{Sh}^{p}(N, v, P)=\frac{1}{100}(20,40,40)$ since $S h_{i}(N, v)=\frac{1}{100}$ for all $i \in N$.

\section{Concluding remarks}

We modeled a situation in which an agent is represented by (possibly) more than one player by a multi-player game. We introduced two solutions for such games. The agent-Shapley value considers the agents in the most unified way in the sense that when an agent enters a coalition, then it enters with all its players. The player-Shapley value takes the players as units, and the payoff to an agent is the sum of the payoffs over all its players.

We gave axiomatic characterizations of these two solutions that differ only in a collusion neutrality axiom. The agent-Shapley value satisfies player collusion neutrality stating that collusion of two players belonging to the same agent does not change the payoff of this agent. On the other hand, the player-Shapley value satisfies agent collusion neutrality stating that after a collusion of two agents, the sum of their payoffs does not change. It is interesting that the possibility for agents to be represented by different players in a multi-player game, makes it possible to have a solution that satisfies such a collusion neutrality axiom as well as efficieny and the null agent property, since for TU-games van den 
Brink (2009) has shown that there is no solution satisfying efficiency, collusion neutrality and the null player property.

We applied both solutions to airport (cost sharing) games, where the agents represent airlines and the players their airplanes. Depending on the type of cost, one of the two solutions introduced in this paper is more suitable. We also applied both solutions to voting games, where the agent-Shapley value is most similar to the 'traditional' ShapleyShubik power index, while the player-Shapley value just assigns weights proportional to the number of seats. This is often used when allocating ministries among government parties.

We conclude by mentioning that in the future we plan to consider more applications of multi-player games. For example, we want to use them to measure centrality or network power in social networks. Traditionally, the nodes in such networks are the positions that an agent or player can occupy in the network. When one agent can occupy (a part of) more than one node at the same time, then we can apply multi-player games to define centrality and power measures for this new setting.

\section{Appendix: Logical independence}

Logical independence of the five axioms stated in Theorem 4.2 is shown by the following alternative solutions.

1. The solution $f_{k}(N, v, P)=0$ for all $k \in M$ satisfies strong monotonicity, symmetry, the null player out property and player collusion neutrality. It does not satisfy efficiency.

2. The solution $f(N, v, P)=N u c\left(v^{P}\right)$ that assigns to every multi-player game the nucleolus of the game $v^{P}$, satisfies efficiency, symmetry, the null player out property and player collusion neutrality. It does not satisfy strong monotonicity.

3. Consider exogenously given positive weights $\omega_{k}>0$, assigned to all agents $k \in M=$ $\{1, \ldots, m\}$. The solution $f_{k}^{\omega}(N, v, P)=\sum_{\substack{H \subseteq M \\ k \in H}} \frac{\omega_{k}}{\sum_{j \in H} \omega_{j}} \Delta_{v}(H)$ that assigns to every multi-player game the weighted Shapley value of $v^{P}$ corresponding to weight system $\omega$, satisfies efficiency, strong monotonicity, the null player out property and player collusion neutrality. It does not satisfy symmetry.

4. The solution $f^{\omega}$ with $\omega_{k}=\left|P_{k}\right|$ that applies the weighted Shapley value with weights equal to the number of players of every agent, satisfies efficiency, strong monotonicity, symmetry and player collusion neutrality. It does not satisfy the null player out property. (Note that this solution itself is not a weighted Shapley value since the weights change if the partition $P$ changes.) 
5. The player-Shapley value satisfies efficiency, strong monotonicity, symmetry and the null player out property. It does not satisfy player collusion neutrality.

Logical independence of the five axioms stated in Theorem 4.3 can be shown by similar alternative solutions. To be precize, the first solution $\left(f_{k}(N, v, P)=0\right.$ for all $\left.k \in M\right)$, also satisfies agent collusion neutrality.

Instead of applying the nucleolus to the game $v^{P}$, consider the solution $f_{k}(N, v, P)=$ $\sum_{i \in P_{k}} N u c_{i}(v)$, that assigns to every agent the sum of the nucleolus payoffs over all its players. This solution satisfies efficiency, symmetry, and agent collusion neutrality, but does not satisfy strong monotonicity.

Considering exogenously given positive weights $\omega_{i}>0$, assigned to all players $i \in N$,

the solution $\bar{f}_{k}^{\omega}(N, v, P)=\sum_{i \in P_{k}} \sum_{\substack{H \subseteq N \\ i \in H}} \frac{\omega_{i}}{\sum_{j \in H} \omega_{j}} \Delta_{v}(H)$ that assigns to every agent the sum of the weighted Shapley values of all its players, satisfies efficiency, strong monotonicity, and agent collusion neutrality. Itt does not satisfy symmetry.

Obviously, the agent-Shapley value satisfies efficiency, strong monotonicity and symmetry, but does not satisfy agent collusion neutrality.

\section{References}

Aumann R.J., And J.H. DrÈze (1974), "Cooperative Games with Coalition Structure", International Journal of Game Theory, 3, 217-237.

Banzhaf, JF (1965) "Weighted Voting Doesn't Work: A Mathematical Analysis", Rutgers Law Review, 19, 317-343.

BRINK, R. VAN DEN (2009), "Efficiency and Collusion Neutrality of Solutions for Cooperative TU-Games", TI-Discussion paper 09/65-1, Tinbergen Institute Amsterdam.

Derks, J.J.M. And H.H. Haller (1999), "Null Players Out? Linear Values for Games with Variable Supports" International Game Theory Review, 1, 301-314.

Graham, D.A., R.C. Marshall, and J.F. Richard (1990), "Differential Payments Within a Bidder Coalition and the Shapley Value", American Economic Review 80, 493-510.

HALler, H. (1994), "Collusion Properties of Values", International Journal of Game Theory, 23, 261-281.

Harsanyi, J.C. (1959), "A Bargaining Model for Cooperative n-Person Games", In Contributions to the Theory of Games IV (eds. Tucker A.W., and R.D. Luce), Princeton UP, Princeton pp 325-355. 
LeHrer, E. (1988), "An Axiomatization of the Banzhaf Value", International Journal of Game Theory, 17, 89-99.

Littlechild, S.C, And G. Owen (1973), "A Simple Expression for the Shapley Value in a Special Case", Management Science, 20, 370-372.

Littlechild, S.C. And G.F. Thompson (1977), "Aircraft landing fees: A game theory approach", The Bell Journal of Economics, 8, 186-204.

MALAWASKI, M. (2002), "Equal treatment, symmetry and Banzhaf value axiomatizations", International Journal of Game Theory, 31, 47-67.

Ni, D., and Y. Wang (2007), "Sharing a polluted river", Games and Economic Behavior 60, 176-186.

Owen, G. (1977), "Values of Games with a Priori Unions", In Essays in Mathematical Economics and Game Theory(eds. Henn R., and O. Moeschlin), Springer Verlag, Berlin pp 76-88.

Shapley, L.S. (1953) "A Value for n-Person Games", In Annals of Mathematics Studies 28 (Contributions to the Theory of Games Vol.2) (eds. Kuhn H.W., and A.W. Tucker), Princeton UP, Princeton pp 307-317.

Shapley, L.S. And M. Shubik (1954), "A Method for Evaluating the Distribution of Power in a Committee System", American Political Science Review, 48, 787-792.

Young, H.P. (1985), "Monotonic solutions of cooperative games", International Journal of Game Theory, 14, 65-72. 